\title{
The Digital Clinic
}

\author{
Doepp Manfred* \\ Holistic Center, Abtwil 9030, Switzerland \\ *Corresponding author: Doepp Manfred, Holistic Center, 13 Haupt St., Abtwil 9030, Switzerland. \\ To Cite This Article: Doepp Manfred. The Digital Clinic. Am J Biomed Sci \& Res. 2021 - 13(6). AJBSR.MS.ID.001927. \\ DOI: 10.34297/AJBSR.2021.13.001927.
}

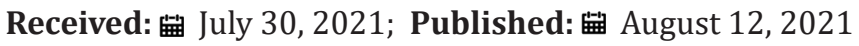

\section{Opinion}

Our future will be digital, or it will not be. That's what you could say if you extrapolate the existing tendencies and trends. Of course, we love the analog world, e.g., that of nature, biology, and all living beings. However, we should have no illusions: in the next few years, the current changes will continue and take more and more serious forms. Artificial Intelligence (AI) is waiting. "1984" will become our reality, a surveillance state, accompanied by a virtual reality, a pseudo-reality. Children will think the virtuality on their screens to be more real than material reality.

To get out of the game, the entire Internet would have to be paralyzed, e.g., because of an electromagnetic pulse "EMP" by a huge solar flare.

These developments also include the official version of a future digital clinic. On a card, a chip, or a cell phone App all information about us will be stored, including data about our behaviour, vaccinations, therapies, and diseases. Something similar is already in place in China. Those who refuse to comply will receive sanctions in the form of loss of freedom and rights.

Those interested in this will be able to keep i.e., our examination results and diagnoses (as well as bank data, cell phone data, fully automated households, self-driving cars, etc.). The complete networking in "Big Data" is imminent and will then be available to every clinic. We can hardly imagine what high-performance computers are already capable of today, and what they can perform as quantum data, and what they will be able to do in the future as quantum computers.

We have contrasted this with our own version of a Digital Clinic.

\section{Topics}

For healthcare, we have two qualifications associated with the timelines existing on earth in our Holistic Center health center. They include the application of the internet and digital communication in medicine, as well as the integration of computerized homeopathy. Homeopathy is accepted and integrated in the health care system in Switzerland.

A patient does not have to appear in person for this, his or her data (including photo or DNA/blood drop) are sufficient to ensure a clear identification.

Quotes from the "Confidential Swiss Letter" dated October 4, 2016, "The Internet is about to begin a new triumph of its technology. From today by 2022, the digital health market will explode to more than 200 billion dollars. With the initiative "Horizon 2020" initiative, the EU is offering 600 million Euros for the digital future of Europe. The digital analysis of health data leads to an individualized medicine for the patient without consulting doctors".

With our methods, we are striving for two goals: a) an individualization and b) an objectification.

Both are not standards in conventional medicine, despite all the Sunday speeches. Instead of individual medical decisionmaking, there are schemes: the so-called evidence-based medicine $=$ EbM Medicine $=\mathrm{EbM}$, based on guidelines; or an irrationality, the so-called authority-based medicine. We want to change this in a positive sense. It is safe to predict that parallel to the transformation of industry into the status 4.0, Information Technology (IT) will also 
take the medicine, as it has control over far more information and its meanings than the brain of a doctor, and much faster. Today's common - rather primitive - medicine of antagonists and blockers will be overtaken by it.

However, the problem remains that the studies on which the IT statements are based are largely influenced or distorted by the pharmaceutical industry. Corruption has also made inroads in this and the Cochrane Collaborations for the control of worldwide scientific publications have a lot of work ahead of them (https:// en.wikipedia.org/wiki/Cochrane_Collaboration).

The ET entity CoBra writes about this: (http://transinformation. net/wann-und-wie-passiert-der-aufstiegpersoenlich-wie-global/): "The pharmaceutical industry was created by the darkness for a few reasons - first, to extract more money from the people who were artificially made sick. Second, to make them [the pharmaceuticals]. in the human bodies to create stress in the biochemical systems, which prevents the raising of the vibration frequency. It is true that certain medicines may help alleviate some symptoms of a particular disease in certain cases, but in most of the cases disease is a manifestation of certain internal blockages, or a manifestation of all the artificial intrusion into the human body. The new Medicine, which is being born on the whole planet right now, raises the vibrational frequency of the human being and the relationship of the human consciousness to the physical body. The key to restoring health and the whole energy here is the plasma level".

\section{What We Do}

At our center, we make cause-related online diagnoses and complete them with online therapies by quantum teleportation. We use the computer-controlled and advanced bioresonance system TimeWaver" (http://en.timewaver.com), which resonates with the individual information patterns of the patient in the global information field.

(https://www.researchgate.net/publication/284186090_ Liouville\%27s_Theorem_in_Classical_Mechanics_and_the_Global_ Information_Field) via a Kozyrev mirror (https://en.scribd.com/ document/184752654/Kozyrev-Mirrors) and two entangled quantum random number generators (https://en.wikipedia.org/ wiki/Hardware_random_number_generator).

The earthly representation of the information field is the Akasha-Chronicle, a holographic quantum field with unlimited capacity. It contains all information concerning the individuals and their souls (deeds, thoughts, feelings, motivations...) of all their incarnations.

The healing themes or measures, which prove to be important for the patient, are transmitted to the subconsciousness as digital frequency patterns, i.e., as a "remote treatment" (https:// de.wikipedia.org/wiki/Quantum_teleportation). This method is independent of space and time.

The patient also receives his results as e-mail attachments, so that he can deal with the contents.

\section{The Information Field}

The basics of the TimeWaver ${ }^{\circ}$ systems about the consciousness and its interaction with matter are found in quantum physics and depth psychology. The connection between the two follows the German physicist Burkhard Heim and his postulated information field (http://archiv.mufonces.org/text/english/heimphysics.htm) and (https://en.wikipedia.org/wiki/Burkhard_Heim). B. Heim developed a 12-dimensional cosmological model. It contains: d1$\mathrm{d} 3$ = matter, $\mathrm{d} 4$ = time, $\mathrm{d} 5$ - $\mathrm{d} 6$ = energetic regulation field, $\mathrm{d} 7-\mathrm{d} 8=$ global information fiel, $\mathrm{d} 9=$ free human will, $\mathrm{d} 10=$ phantasies, $\mathrm{d} 11$ $=$ cosmic consciousness, and $\mathrm{d} 12=$ the source (Figure 1 ).

\section{Digital Homeopathy}

No medical field can be better optimized by that than homeopathy because it is systematized and works on the information level (d7). In it, the diagnostic basis is the repertorization procedure (https://de.wikipedia.org/wiki/Arndt\%E2\%80\%93Schulz_rule) and (https://de.wikipedia.org/wiki/Samuel_Hahnemann). Repertorium-Software can be used to facilitate the search for the identity of the simile: https://www.softguide.de/programm/ radaropus-easy-homoeopathie-software.

However, the software of the TimeWaver $^{\circ}$ is not limited to homeopathy but contains more than 1,000,000 digitalized information about pathologies on the one hand and remedies on the other. Their correspondences and themes on the levels d1 to d8 can be identified.

\section{Critics and Limitations}

In the field of testing methods, there is generally an antagonism between sensitivity and stability: the more sensitive a method, the less stable or reproducible it is. The here described surveys of the information field are very sensitive. To guarantee the necessary degree of reproducibility, one criterion must be added: the plausibility. It should be given by the user who sorts out improbable results based on his experience and demands repetitions in case of unclear results.

It is therefore also clear that this system could be fully system could be fully automated, but this should not be aimed at since there are weaknesses about reproducibility. The consciousness of the human being with its openness to intuition, conscience and ethics is irreplaceable. 




Figure 1: The 12 dimensions of Burkhard Heim.

This is what the producers of artificial intelligence devices have also had to realize this. The information field is not a simple computer memory but is subject to the cosmic-divine laws. Egocentric questions, which contradict the divine laws, are answered in absurd and/or wrong way.

An example: one could find out the upcoming lottery numbers with the help of the yes/no function. This is completely egocentric; the results will be wrong. If a user similarly lets his expectations or doubts flow in, the results will be inadequate, as well as if an IT-only program is active.

A crucial point is therefore that the user "human" has control over questions and answers, that he is neutral and has a stable consciousness. Only then the information field answers and works optimally and can guarantee a high accuracy rate. Misuse of the system can thus not be ruled out, which, however, unfortunately applies to almost all human knowledge, techniques, and technologies.

\section{The Future}

It fulfils the expectation and description of the $6^{\text {th }}$ KondratieffWave cycle (http://www.kondratieff wavecycle.com/kondratieff -wave) for the period of the integration of IT in the individual health care. Wikipedia: "Several candidates have been identified as technologies that dominate the $6^{\text {th }}$ Kondratieff -cycle: Biotechnology, Nanotechnology, psychosocial health and competence, mobile Internet ...".

As early as 1996, Nefedov, in his book "The Sixth Kondratieff " argued that the next fundamental innovation will take place in healthcare. (https://www.goodreads.com/author/show/4173368. Sergey_A_Nefedov).

This is a version of the medicine of the future that we use successfully in everyday life, and we present it here. It is a gentle, holistic medicine of vibrations and by means of vibrations/ digital frequency patterns, which integrates modern information technological innovations.

Of course, it cannot replace symptom-related emergency medicine or surgical interventions, but the emphasis is on finding and treating the causes of diseases, preferably as prophylaxis and prevention. 